\title{
ROB01 protein expression is independently associated with biochemical recurrence in prostate cancer patients who underwent radical prostatectomy in Asian patients
}

\author{
Sang Hoon Kim ${ }^{1}$, Tae-Jung Kim², Dongho Shin ${ }^{3}$, Kyung Jae Hur ${ }^{3}$, Sung-Hoo Hong ${ }^{3}$, Ji Youl Lee ${ }^{3}$, \\ U-Syn $\mathrm{Ha}^{3,4}$
}

${ }^{1}$ Department of Urology, Eunpyeong St. Mary's Hospital, College of Medicine, The Catholic University of Korea, Seoul, Republic of Korea; ${ }^{2}$ Department of Hospital Pathology, College of Medicine, The Catholic University of Korea, Seoul, Republic of Korea; ${ }^{3}$ Department of Urology, Seoul St. Mary's Hospital, College of Medicine, The Catholic University of Korea, Seoul, Republic of Korea; ${ }^{4}$ Cancer Research Institute, College of Medicine, The Catholic University of Korea, Seoul, Republic of Korea

Contributions: (I) Conception and design: SH Kim; (II) Administrative support: US Ha; (III) Provision of study materials or patients: TJ Kim, DH Shin, KJ Hur, SH Hong, JY Lee; (IV) Collection and assembly of data: SH Kim; (V) Data analysis and interpretation: SH Kim, US Ha; (VI) Manuscript writing: All authors; (VII) Final approval of manuscript: All authors.

Correspondence to: U-Syn Ha, MD, PhD. Department of Urology, Seoul St. Mary's Hospital, College of Medicine, The Catholic University of Korea, 222, Banpo-daero, Seocho-gu, Seoul 06591, Republic of Korea. Email: ushamd@catholic.ac.kr.

Background: The purpose of this study is to investigate the correlation between ROBO1 expression and prostate cancer aggressiveness.

Methods: ROBO1 expression was evaluated in normal prostate epithelial cells (PrEC) and different prostate cancer cell lines by Western blot analysis. The migration and invasion of native and ROBO1 knockdown cells were evaluated using migration chambers and a Matrigel-coated membrane, respectively. Samples from 145 patients who underwent radical prostatectomy between June 2000 and June 2008, were retrieved from the paraffin files for tissue microarray (TMA) with immunohistochemical analysis. Biochemical recurrence (BCR)-free survival curves were estimated using the Kaplan-Meier and Cox regression methods in two groups of patients classified according to the degree of ROBO1 expression (low or high expression).

Results: ROBO1 is highly expressed in the prostate cancer cell lines. All ROBO1 knockdown cells (PC3, $22 \mathrm{Rv} 1$ and DU 145) showed markedly decreased migration and invasiveness compared to native cells. In 145 patients with radical prostatectomy, the Kaplan-Meier curves and log-rank test for BCR-free survival stratified by ROBO1 expression in organ-confined (pT2) or not (pT3), showed significant differences in 10-year survival between the ROBO1 high and low expression groups $(87.2 \%$ versus $52.6 \%$ in pT2; $\mathrm{P}=0.047,51.0 \%$ versus $36.9 \%$ in pT3; $\mathrm{P}=0.033$ ). The multivariable-adjusted model showed a markedly increased hazard ratio (HR) in patients with high ROBO1 expression compared to the patients with low ROBO1expression in every model.

Conclusions: ROBO1 may play an important role in the migration and invasion of prostate cancer cells, and was independently associated with BCR.

Keywords: Prostate cancer; ROBO1; biochemical recurrence (BCR); radical prostatectomy

Submitted Jun 19, 2021. Accepted for publication Sep 03, 2021.

doi: $10.21037 / g s-21-406$

View this article at: https://dx.doi.org/10.21037/gs-21-406

^ ORCID: 0000-0001-9930-0588. 


\section{Introduction}

Prostate cancer is a heterogeneous disease and presents a diverse disease course. The intricate nature of prostate cancer makes it difficult to predict the prognosis and natural course of the disease. This heterogeneity also makes it difficult for physicians to manage the disease. Especially, the principal problem arising from prostate cancer is local invasion and metastasis, and it is hard to predict prostate cancer aggressiveness based on the established staging system.

In acquiring cancer cell aggressiveness, genotypical changes occur that break the prostatic tissue barriers, allowing the primary lesion to escape and establish metastatic tumors (1). The key to optimizing prostate cancer management requires a comprehensive understanding of the molecular factors that underlie prostate cancer progression. However, there is insufficient information regarding the molecular mechanisms that drive prostate cancer progression. Recently, accumulated evidence indicated that signaling pathways involved in the development were altered in tumorigenesis (2). ROBO1 is a member of the roundabout (ROBO) immunoglobulin superfamily of proteins (3). Recent studies showed that the ROBO1 protein plays a crucial role in cell motility and migration during embryogenesis and organogenesis $(4,5)$. In addition, evidence showed that ROBO1 might drive migration and invasion in malignant cells, such as glioma and breast cancer $(6,7)$, which might play a role in cancer aggressiveness. In contrast, some studies suggested that ROBO1 pathways play a key role in tumors by acting as a tumor suppressor, especially in cell invasion $(8,9)$. Regarding prostate cancer, studies have only focused on comparative expression analysis between normal and prostate cancer, not on its clinical significance in prostate cancer patients $(10,11)$.

The purpose of this study was to clarify the correlation of ROBO1 expression with prostate cancer aggressiveness and prognosis. In this study, we investigated the clinical significance of ROBO1 expression in an in vitro study, which addressed the effect of $\mathrm{ROBO} 1$ on the aggressiveness of prostate cancer using prostate cancer cell lines.

We present the following article in accordance with the REMARK reporting checklist (available at https://dx.doi. org/10.21037/gs-21-406).

\section{Methods}

\section{Cell culture}

A normal prostate cell line, RWPE-1 (ATCC, Manassas, VA, USA), and human prostate cancer DU145, PC-3, and
LNCap (Korean Cell Line Bank, Seoul, Korea) cell lines were cultured in RPMI 1640 supplemented with $10 \%$ fetal bovine serum (FBS), L-glutamine $(300 \mathrm{mg} / \mathrm{L}), 25 \mathrm{mM}$ HEPES, and $25 \mathrm{mM} \mathrm{NaHCO}_{3}$. The cells were incubated at $37{ }^{\circ} \mathrm{C}$ with $5 \% \mathrm{CO}_{2}$. The shRNA (gene knockdown) target sequences $\left(5^{\prime}-3\right.$ ') were as follows: negative control targeting LacZ, AATTTAACCGCCAGTCAGGCT; human LCN2, GGAGCTGACTTCGGAACTAAA; and human SLUG, CAGC TGTAAATACTGTGACAA.

\section{Immunoblotting}

The cells were harvested, washed twice with phosphatebuffered saline (PBS) and lysed in RIPA buffer that contained $25 \mathrm{mM}$ Tris $\mathrm{HCl}(\mathrm{pH}$ 7.6), $150 \mathrm{mM} \mathrm{NaCl}$, $1 \%$ NP-40, $1 \%$ sodium deoxycholate, and $0.1 \%$ sodium dodecyl sulfate (SDS) supplemented with a protease inhibitor cocktail (Roche, Grenzacherstrasse, Base, Swiss). The lysed cells were centrifuged at $13,000 \mathrm{rpm}$ at $4{ }^{\circ} \mathrm{C}$ for 15 minutes, and the supernatant was collected and stored at $-75{ }^{\circ} \mathrm{C}$ for further analysis. The protein extracts $(20 \mu \mathrm{g})$ were electrophoresed on 6\% SDS-polyacrylamide gels, and the proteins were then transferred to a polyvinylidene fluoride membrane (Immobilon-P; Millipore, Billerica, MA, USA). After transfer, the membrane was blocked in $5 \%$ skim milk for 30 minutes and then reacted with primary antibodies specific for ROBO1 (dilution, 1:1,000; Millipore) and incubated at $4{ }^{\circ} \mathrm{C}$ for 16 hours. The membrane was washed with TBS-T $(10 \mathrm{mM}$ Tris, $150 \mathrm{mM}$ sodium chloride, $\mathrm{pH} 7.6$, and $0.1 \%$ Tween 20 ) and incubated with secondary anti-mouse antibodies (dilution, 1:2,000; Invitrogen Corporation, Paisley, UK) conjugated to horseradish peroxidase for 30 minutes. The reactions were finally analyzed using a chemiluminescence detection system (Thermo Scientific, Rockford, IL, USA). We used $\beta$-actin (Santa Cruz Biotechnology, Dallas, TX, USA) as an internal control for protein loading.

\section{Migration and invasion assay}

Native prostate cancer cells (DU145, PC-3, and LNCap) and knockdown cells (DU145/shROBO1, PC3/shROBO1, LNCap/shROBO1) were examined for cell motility using migration chambers and tested by the ability of the cells to invade through a Matrigel-coated membrane. Briefly, the cells were seeded in the top chamber of $8.0 \mu \mathrm{m}$ pore-sized cell culture inserts that were either coated or uncoated with Matrigel for migration and invasion assays, respectively. 
Then, the inserts were placed in a 24-well plate filled with medium with 5\% FBS. After $24 \mathrm{~h}$, the cells that penetrated to the underside surface of the inserts were fixed and stained with Diff-Quick (Fisher Scientific, Pittsburgh, PA, USA) and counted and compared to native prostate cancer cells and knockdown cells under light microscopy. The mean number of cells counted in three high-power fields for each condition in triplicate samples was calculated.

\section{Clinical outcomes}

The analysis was conducted on patients who underwent radical prostatectomy between June 2000 and June 2008. To eliminate the influence of the surgical margin status and adjuvant therapy, we included only 145 patients who had a final pathologic diagnosis of pT2 to pT3a adenocarcinoma without surgical margin involvement, and none of these patients had undergone preoperative or adjuvant therapy of any type. The study was conducted in accordance with the Declaration of Helsinki (as revised in 2013). The study was approved by the institutional review board (IRB) of the Catholic University of Korea (No. KC15SIS10600). Informed consent was waived due to the retrospective nature of the study. The mean patient age at the time of surgery was $63.5 \pm 6.5$ years (range, $39-77$ years). The hematoxylin and eosin-stained slides were independently reviewed by two pathologists (TJK and YJC) in each case to confirm the original diagnosis. The Gleason scores were categorized into Gleason scores of 4-6 (GS $\leq 6)$, a Gleason score of $7(\mathrm{GS}=7)$, and a Gleason score of 8-10 (GS $\geq 8$ ), as recommended previously (12). The follow-up, which included a prostate-specific antigen (PSA) test, was conducted every 3-6 months or more frequently if the PSA was rising from the nadir. All 145 radical prostatectomy cases were retrospectively retrieved from the paraffin files for tissue microarray (TMA) with immunohistochemical analysis.

\section{$T M A$}

The TMA recipient blocks were constructed containing the most representative cores of paraffin-embedded prostate adenocarcinoma tissues and paired normal prostate tissue cores from each 145 archival patient specimens, which were previously fixed in $10 \%$ formaldehyde according to established methods. The least differentiated tumor area was selected for TMA. From every archival paraffin block, one cylinder of $2.0 \mathrm{~mm}$-diameter tissue was taken from representative areas and transferred to the paraffin recipient blocks using a Quick-Ray ${ }^{\circledR}$ Tissue Microarrayer (UNITMA, Seoul, Korea). Four cores were sampled and included in the TMA for each patient. The control cores consisting of normal tonsil tissue, normal lung tissue, normal colonic mucosa, and basal cell carcinoma were included in every TMA block.

\section{Immunobistochemistry}

The TMA blocks were cut into $4 \mu \mathrm{m}$-thick serial sections and mounted on silanized glass slides. After deparaffinization, heat-induced epitope retrieval was conducted by immersing the slides in Coplin jars filled with $10 \mathrm{mmol} / \mathrm{L}$ citrate buffer (Ph 6.0) and boiling in a model RHS-1 microwave vacuum histoprocessor (Milestone, Bergamo, Italy) at a controlled final temperature of $121{ }^{\circ} \mathrm{C}$ for $15 \mathrm{~min}$. After epitope retrieval, the slides were treated with $3 \% \mathrm{H}_{2} \mathrm{O}_{2}$ in methanol for $10 \mathrm{~min}$ at room temperature to abolish endogenous peroxidase activity. Next, the slides were incubated with anti-rabbit polyclonal ROBO1 antibody (1:100, cat\# ab7279, Abcam, Cambridge, MA, USA) or anti-rabbit polyclonal Gli-1 antibody (1:30, cat\# sc20687, Santa Cruz Biotechnology) using a Ventana Benchmark Ultra autostainer (Roche A/S, Hvidovre, Denmark) with an OptiView detection kit.

The immunostaining was interpreted as positive when cytoplasmic staining for ROBO1 was evident, according to the literature. The positive controls for ROBO1 were bronchial epithelial cells and colonic mucosal cells. For the negative controls, PBS was used instead of the primary antibodies. The immunostaining and the histology were interpreted by two pathologists (TJK and YSC). The ROBO1 staining intensity was semi-quantified and scored into four categories: 0 , no positive cells; $1+, 1 \%$ to $10 \%$ positive cells; $2+, 11 \%$ to $50 \%$ positive cells, and $3+$, more than $50 \%$ positive cells. Then, the cases were subdivided into low expression $(0$ and $1+)$ and high expression groups $(2+$ and $3+)$. Figure 1 shows the expression of ROBO1 in patients with prostate cancer.

\section{Oncological outcomes}

The oncologic outcome of BCR-free survival was obtained from the medical charts and radiographic reports. PSA levels of $>0.20 \mathrm{ng} / \mathrm{mL}$ by two subsequent measurements 30 days after radical prostatectomy were defined as BCR (13). Follow-up PSA data were assessed in all cases and there were 

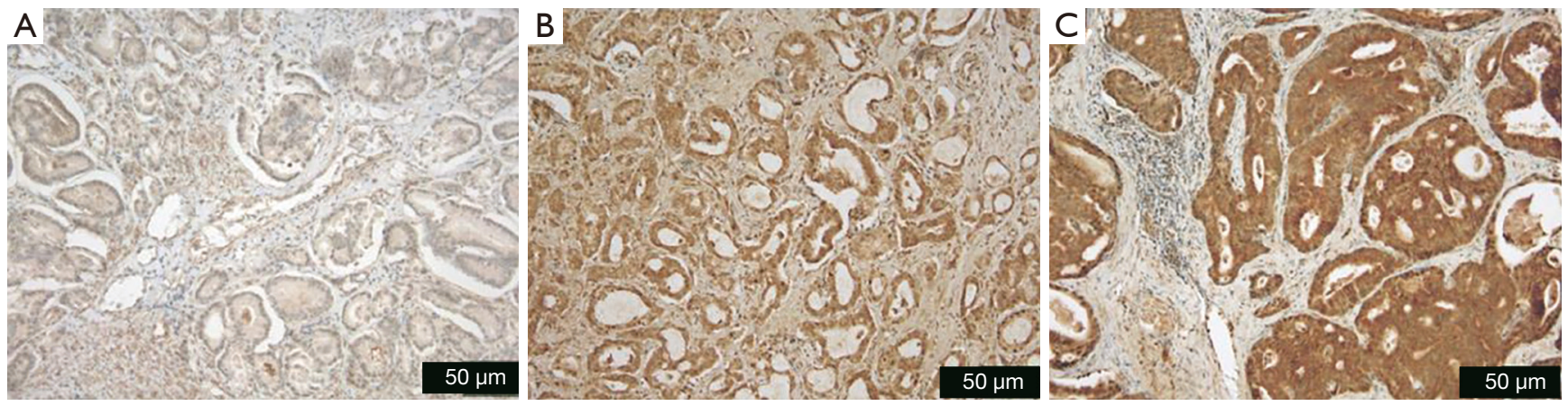

Figure 1 Immunohistochemical staining of ROBO1 expression in prostate cancer: (A) ROBO1-negative, (B) ROBO1 low expression, and (C) ROBO1 high expression $(\times 200)$.

no deaths that occurred before BCR. BCR-free survival was defined as the duration from radical prostatectomy to BCR. Before BCR, no treatment including radiation or hormone therapy was performed on any patient.

The data obtained by the above-mentioned method were analyzed and compared with the corresponding data in the two subgroups classified according to the degree of ROBO1 expression (low or high expression). The data for the study are expressed as the frequency and means \pm the standard deviations of the means in the patients with radical prostatectomy. Comparisons of the two groups were made using a $\chi^{2}$ test or an independent Student's $t$-test. BCRfree survival curves were estimated using the Kaplan-Meier method. The survival curves were compared using the log-rank test. To determine whether ROBO1 expression affected oncological outcomes, Cox proportional hazard models were constructed with adjustment for certain factors, including T-stage (T2, T3a), Gleason score, PSA, age, and tumor volume, resulting in models 1,2 , and 3 .

\section{Statistical analysis}

Statistical analyses were performed using SPSS for Windows version 11.50 statistical software (SPSS, Chicago, IL, USA). All experiments were performed on three separate cultures. All data are presented as the mean \pm standard deviation, where $\mathrm{P}<0.05$ was considered statistically significant. The overall comparisons between the groups were performed using the SPSS program (version 12.0). The adjusted odds ratio of ROBO1 expression was calculated using ROBO1 low expression as the reference and were judged at a significance level of $\mathrm{P}<0.05$ in a forward-conditional stepwise logistic regression analysis of the independent parameters.

\section{Results}

\section{ROBO1 is highly expressed in prostate cancer cell lines}

ROBO1 protein expression was examined in normal prostate cells (RWPE-1) and different prostate cancer cell lines by Western blot analysis. ROBO1 was not expressed in RWPE-1 cells and was highly expressed in DU145, PC-3, and LNCap cells. Figure 2 shows the protein expression in stably transduced knockdown cells compared to native PC3 cells by Western analysis. The blots show distinct ROBO1 knockdown in PC3 native cells, and also distinct ROBO1 knockdown in the DU 145 and LNCap cells.

\section{ROBO1 plays an important role in the aggressiveness of prostate cancer cells}

The migration of $\mathrm{PC} 3 / \mathrm{shROBO} 1$ cells was decreased markedly compared to native PC-3 cells (Figure $3 A$ ). This decreasing pattern of migration was also seen in DU145/ shROBO1 and LNCap/shROBO1 cells compared to native DU145 and native LNCap cells. ROBO1 knockdown markedly decreased cancer cell migration in invasiveness testing of the cancer cells. All ROBO1 knockdown prostate cancer cells (PC3, DU 145, and LNCap) showed markedly decreased invasiveness compared to the native cells (Figure 3B).

\section{Association of ROBO 1 protein expression with clinicopathologic parameters}

High ROBO1 expression in tumor cells was detected in $25.8 \%(39 / 145)$ of the patient samples. Table 1 shows the distribution of protein expression according to clinicopathologic parameters. The expression of 
A
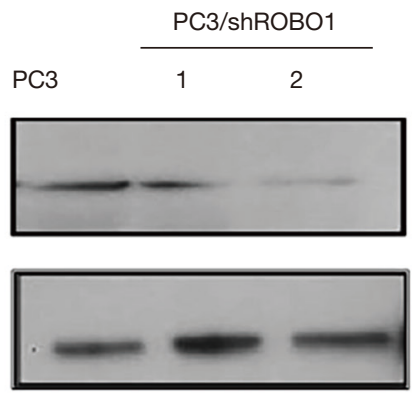

B

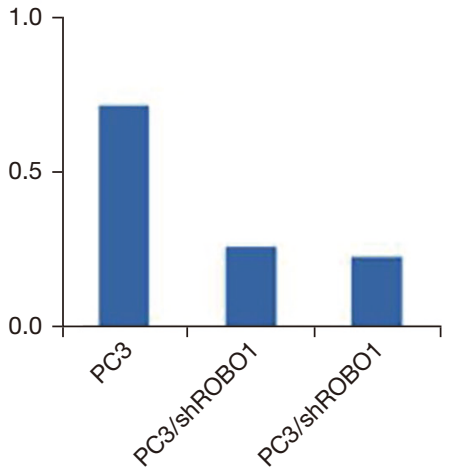

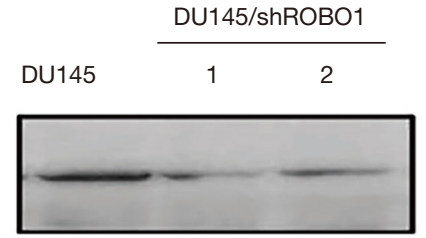
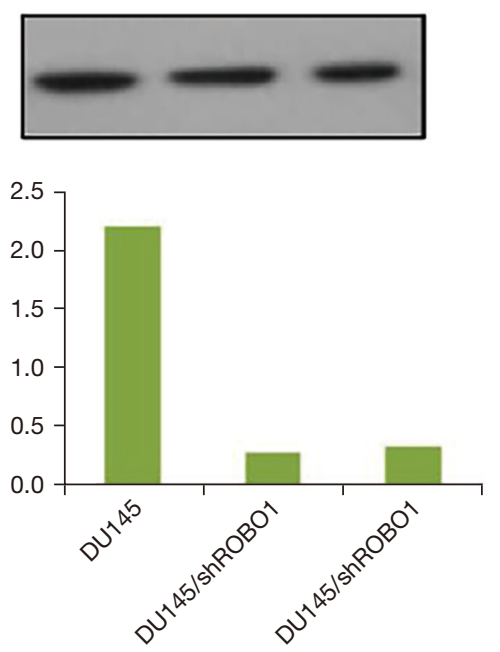
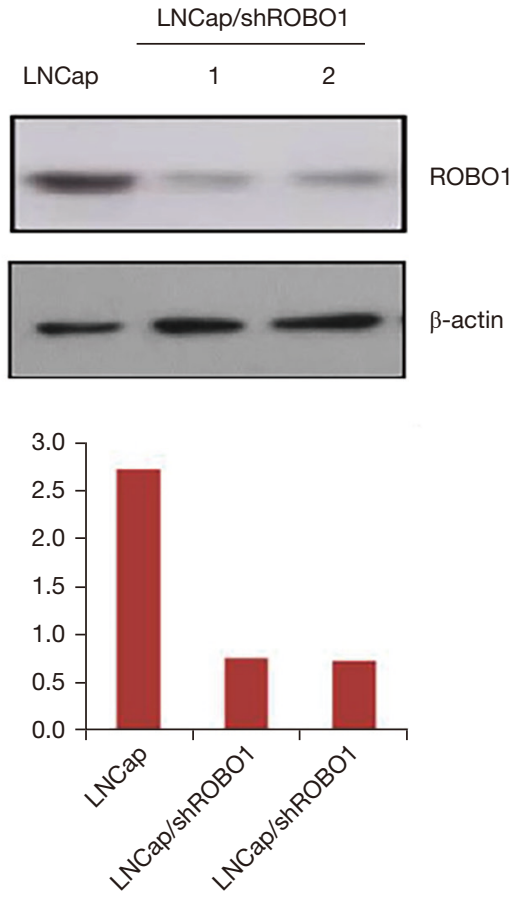

Figure 2 The expression of ROBO1 in prostate cancer cell lines. (A) Western blot analysis and (B) densitometric analysis relative to $\beta$-actin. Western analysis shows ROBO1 protein expression examined in different prostate cancer cell lines and knockdown cells. The expression of ROBO1 was distinctly different between the native with PC-3, DU145, and LNCap knockdown cells.

ROBO1 protein was not significantly associated with clinicopathologic parameters, including age, prostate size, tumor size, pretreatment PSA, T-stage, and Gleason score. Only lymph node metastasis reached statistical significance.

\section{Association of ROBO 1 expression with BCR-free survival}

The mean follow-up period for patients with high ROBO1 expression was 85 months (range, 4-155 months; median 79) and 88 months (range, 3-153 months; median, 81 ) in low ROBO1-expressing patients. In 145 patients with radical prostatectomy, $37(25.5 \%)$ pT2 patients showed BCR at the last follow-up, in which BCR was seen in $6(30.0 \%)$ patients with high ROBO1expression and 3 (5.9\%) patients with low ROBO1 expression. BCR was seen 10 (52.6\%) of the pT3 high ROBO1-expressing and 18 (32.7\%) of the low ROBO1-expressing patients. The Kaplan-Meier probability of BCR-free survival stratified by ROBO1 high or low expression in organ-confined (pT2) or not (pT3) disease is shown in Figure $4 A, 4 B$, respectively. The 10-year BCR-free survival of pT2 patients was $87.2 \%$ in the ROBO1 low-expressing patients and $58.4 \%$ in the ROBO1 high-expressing patients, and a significant difference was found by the log-rank test between the two groups. In the pT3 patients, the 10-year BCR-free survival was $51.0 \%$ in the low ROBO1 expression group and $36.9 \%$ in the high ROBO1 expression group, and a significant difference was also found between the two groups.

Table 2 shows the hazard ratio (HR) for BCR stratified according to high and low expressions of ROBO1 in T-stage-adjusted and multivariable-adjusted model 2.3. A marked increase in HR in patients with a high expression of ROBO1 was seen compared to the ROBO1 lowexpressing patients in every model. The multivariate analysis demonstrated the high expression of ROBO1 as an independent prognostic factor for BCR.

\section{Discussion}

The main findings of this study were: (I) ROBO1 may play an important role in the migration and invasion of prostate cancer cells; (II) high expression of ROBO1 was associated with a significantly higher risk of BCR; and (III) ROBO1 could be an independent prognostic factor for BCR.

The role of ROBO 1 in cell migration during organogenesis is well established. Dysregulation of the 

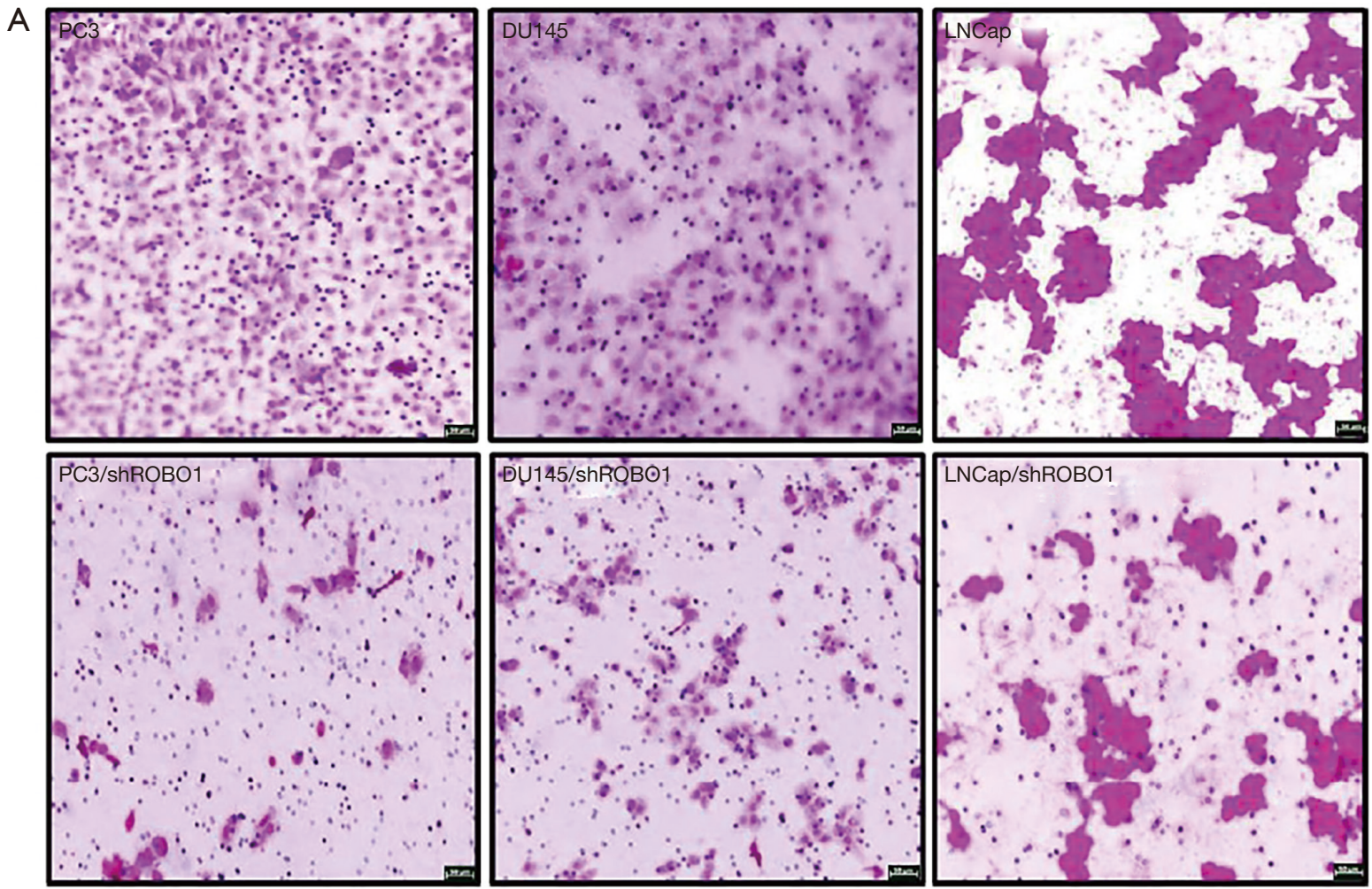

B
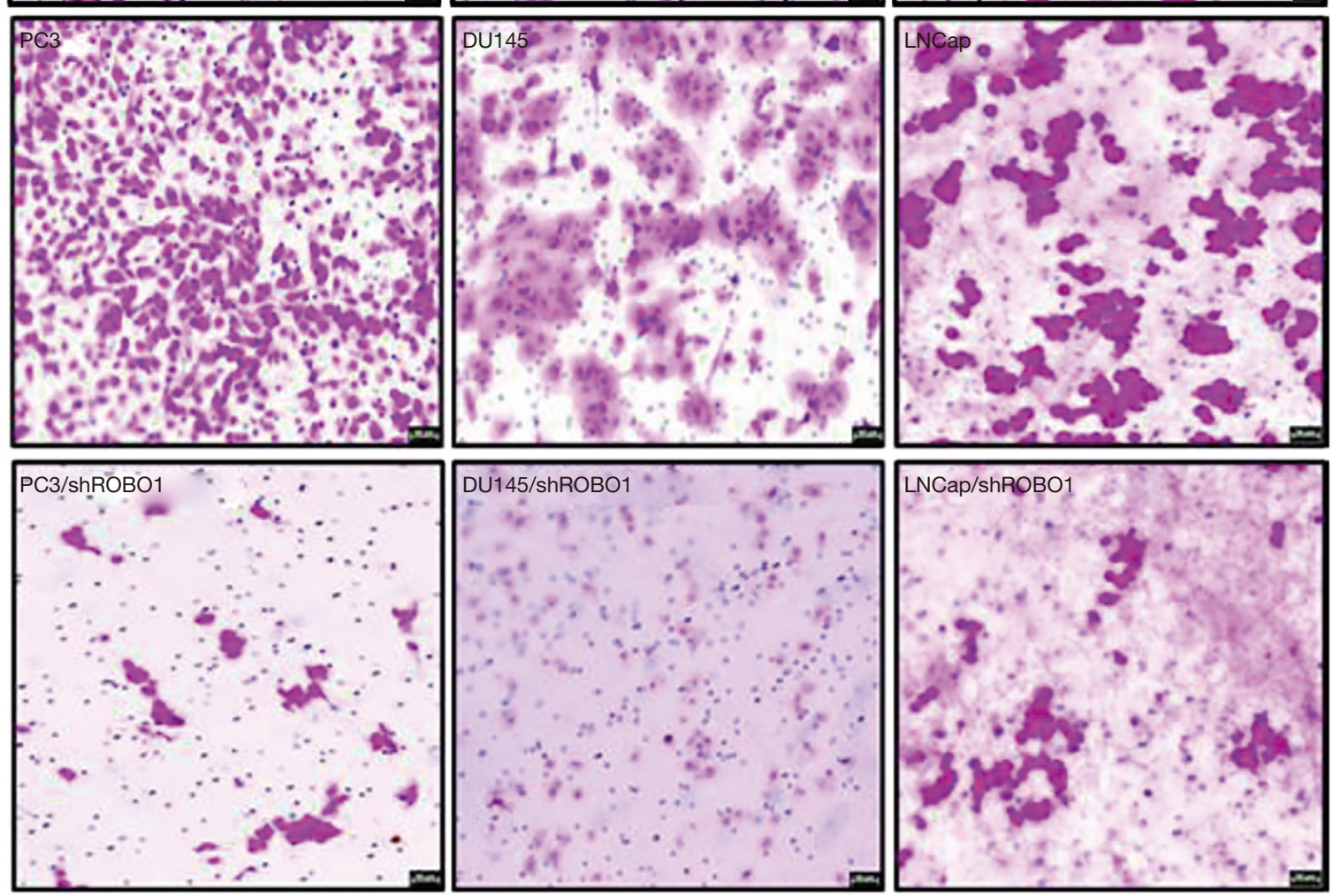

Figure 3 Migration and invasion ability in native prostate cancer cell lines compared to ROBO1 knockdown cells ( $\times 100)$. (A) Native prostate cancer cells and ROBO1 knockdown cells were examined for cell motility using migration chambers; (B) native prostate cancer cells and ROBO 1 knockdown cells were examined for cell invasiveness, tested using a Matrigel-coated membrane. 
Table 1 Distribution of ROBO1 protein expression in 145 patients who underwent radical prostatectomy

\begin{tabular}{|c|c|c|c|}
\hline Variable & \multicolumn{2}{|c|}{ ROBO 1 expression } & $P$ value \\
\hline Age (years) & & & 0.141 \\
\hline$\leq 60$ & $32(22.1)$ & $7(4.8)$ & \\
\hline$>60$ & $74(51.0)$ & $32(22.1)$ & \\
\hline$<30$ & $22(15.2)$ & $6(4.1)$ & \\
\hline$\geq 30$ & $84(57.9)$ & $33(22.8)$ & \\
\hline Tumor size $\left(\mathrm{cm}^{3}\right)$ & & & 0.878 \\
\hline$<5$ & $64(44.1)$ & $23(15.9)$ & \\
\hline$<20$ & $95(65.5)$ & $36(24.8)$ & \\
\hline$\geq 20$ & $11(7.6)$ & $3(2.1)$ & \\
\hline pT classification & & & 0.735 \\
\hline pT2 & $51(35.2)$ & $20(13.8)$ & \\
\hline pT3 & 55 (37.9) & $19(13.1)$ & \\
\hline Gleason grade & & & 0.624 \\
\hline$\leq 6$ & $61(42.1)$ & $19(13.1)$ & \\
\hline 7 & $35(24.1)$ & $16(11.0)$ & \\
\hline
\end{tabular}

ROBO pathway has a role in oncogenesis that is associated with the development, migration, and invasiveness of cancer cells $(14,15)$. It has been suggested that ROBO1 could be involved in the tumorigenesis of several solid tumors including breast (16), lung (17), ovary (18), cervical (19), and liver cancer (20), and reports indicate that the Slit/ROBO pathways differentially modulate invasion and migration. Research on the relationship between the pathogenesis of cancer and the ROBO1 pathway showed contrary roles in the cancer development process depending upon the type of cancer. These contrary actions in the cancer process might depend upon the type of cancer and signaling, although they have not yet been explained.

Little is known about the role of ROBO1 in prostate cancer. There are only two studies reported on prostate cancer patients. Although two studies suggested that Low expression of ROBO1 is associated with poor survival, It is noteworthy that such results are only outcome from African-American patients. On the contrary, such result was not relevant with Caucasian patients, which may be influence by ethnic biological differences.

Parray et al. showed that ROBO1 negatively regulates motility and the invasiveness of primary prostate cancer cells, and its loss causes these cells to acquire invasive traits (21). Ferrari et al. (22) reported that loss of $R O B O 1$ causes disintegration of the DOCK1 complex that in turn triggers invasiveness of cancer cells through loss of E-Cadherin and activation of Rac1 signaling However, our results were 

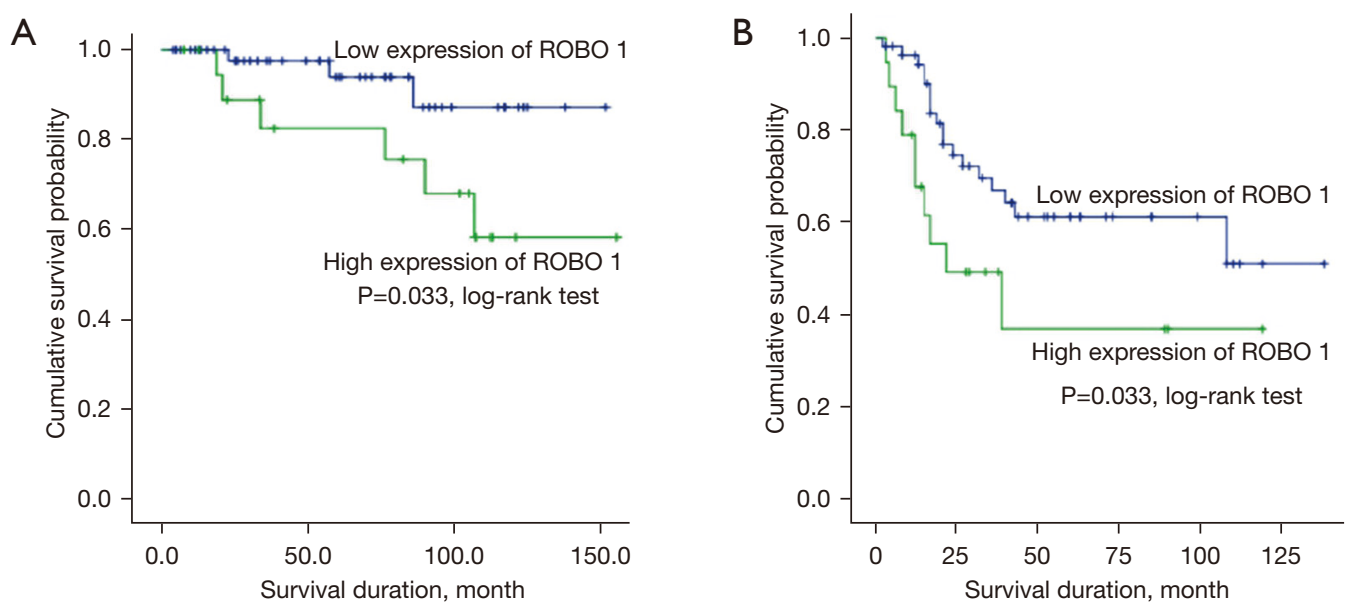

Figure 4 Kaplan-Meier curve shows survival probability for (A) pT2 and (B) pT3 patients according to ROBO1 expression.

Table 2 T-stage and multivariable-adjusted hazard ratios for biochemical recurrence according to the expression of ROBO1

\begin{tabular}{|c|c|c|c|c|c|c|c|c|c|}
\hline ROBO1 & \multicolumn{3}{|c|}{ Model 1* } & \multicolumn{3}{|c|}{ Model $2^{\star *}$} & \multicolumn{3}{|c|}{ Model $3^{\star \star \star}$} \\
\hline Low & Ref. & - & - & Ref. & - & - & Ref. & - & - \\
\hline High & 2.661 & $1.326-5.340$ & 0.006 & 2.504 & $1.261-4.972$ & 0.009 & 2.448 & $1.255-4.933$ & 0.009 \\
\hline
\end{tabular}

*, adjusted by T-stage; ${ }^{\star \star}$, adjusted by T-stage, Gleason score, and PSA; ${ }^{* \star}$, adjusted by T-stage, Gleason score, PSA, age, and tumor volume. The data are presented as HRs (95\% confidence interval). HR, hazard ratio; Cl, confidence interval; PSA, prostate-specific antigen.

different from those of Parray's. The present study showed that the knockdown of ROBO1 expression in prostate cancer cells resulted in a decreased capacity for migration and invasiveness. And we demonstrated that these in vitro results correlated with clinical survival by analyzing clinical data with over 10-year follow-ups, which showed that the patients in the same stage with high ROBO1 expression had a higher probability for recurrence and poor prognosis. Although the contradictory results in the scanty knowledge for the role of ROBO1 in prostate cancer cannot be explained, the current results could be partially explained by a few studies that showed ROBO1 expression is significantly associated with an increased metastatic risk through vasculogenesis, angiogenesis, and lymphangiogenesis $(23,24)$. ROBO signaling induced and promoted micrometastasis, and poorer prognosis. Based on these results, including the current results, we propose that ROBO1 signaling may be inversely correlated with prognosis.

Another possible reason could be ethnic differences. Generally, prostate cancer has different characteristics in each race. It may be assumed that these different ethnic characteristics may affect the role of ROBO1. A previous study comparing Caucasians and African-Americans showed ethnic differences in ROBO1 expression in prostate cancer tissue (21). In African-Americans with prostate cancer, significant differences in ROBO1 expression were seen in the progression of prostate cancer. In contrast, differences in $\mathrm{ROBO} 1$ expression were not seen in the progression of prostate cancer in Caucasians.

In our study, ROBO1 expression was analyzed with respect to the T stage and Gleason grade. For both clinical factors, an increase in $\mathrm{ROBO} 1$ expression was not observed as the stage and Gleason grade advanced. These are seemly paradoxical findings for the biological role of ROBO signaling, where ROBO 1 expression could be differ from according to expected stage, considering the generally known information that a dysregulation in ROBO plays a role in cancer cell migration and invasion. Consistent with our results, a study of ROBO1 expression in gallbladder cancer showed no significant difference in ROBO1 immunoreactivity with respect to localized (pT2) and advanced $(\mathrm{pT} 3,4)$ gallbladder cancer $(25)$. This study 
indicated that ROBO1 expression was inversely correlated with overall survival, suggesting ROBO1 signaling as a poor prognostic factor for micro-metastasis. We can hypothesis that these biological roles of ROBO1 to promote micrometastasis affect the prognosis of prostate cancer, without influencing the gross stage and tumor grade.

The distinctive feature of our study was that, to the best of our knowledge, it is the first study in Asian patients to perform a comparative analysis according to ROBO1 expression on oncological survival. Previous study conducted by Parray et al. (21) focused on the crosssectional studies of the relationship with pathologic stage or in vitro studies. Current study suggested the clinical significance of ROBO1 expression based on the long-term oncological analysis. Comparing with the results of Ferrari et al. (22), current analysis through the stratified stage and relatively large numbers of patients might be a distinction.

It is hard to decide the clinical significance based only on in vitro studies or pathologic findings without oncological survival analysis. Therefore, the present study was conducted to evaluate the correlation with clinical results of genetic analyses to suggest the prognostic significance of ROBO1 expression.

Here, we applied multivariate linear regression analysis to adjust possible prognostic factors including T-stage, Gleason score, PSA, age, and tumor volume. The final regression model was comprised of risk factors for BCR, indicating that ROBO1 expression was significantly correlated with BCR.

There are some limitations to the generalizability of these results. First, we cannot exclude selection bias because all of the enrolled patients were suitable for radical prostatectomy. Generally, surgical indications are limited to clinically localized or minimally advanced stages and patients who show evidence of metastasis in preoperative staging evaluation cannot be included. Thus, this limited the subjects for analysis and could have imparted selection bias. However, based on these observations, we might propose that ROBO1 expression plays a role in the poor prognosis of localized prostate cancer.

\section{Conclusions}

This study objectively demonstrated the clinical significance of ROBO1 expression in correlation with an in vitro study. An increased expression of $\mathrm{ROBO} 1$ protein could indicate a significantly higher risk of BCR. Thus, these results may suggest ROBO1 as a useful prognostic biomarker in prostate cancer for determining the likelihood of BCR.

\section{Acknowledgments}

Funding: This work was supported by the National Research Foundation of Korea (NRF) grant funded by the Korea government (No. NRF-2018R1D1A1B07049542).

\section{Footnote}

Reporting Checklist: The authors have completed the REMARK reporting checklist. Available at https://dx.doi. org/10.21037/gs-21-406

Data Sharing Statement: Available at https://dx.doi. org/10.21037/gs-21-406

Conflicts of Interest: All authors have completed the ICMJE uniform disclosure form (available at https://dx.doi. org/10.21037/gs-21-406). The authors have no conflicts of interest to declare.

Ethical Statement: The authors are accountable for all aspects of the work in ensuring that questions related to the accuracy or integrity of any part of the work are appropriately investigated and resolved. The study was conducted in accordance with the Declaration of Helsinki (as revised in 2013). This study was approved by the Institutional Review Board (IRB) of the Catholic University of Korea (IRB No. KC15SIS10600), and individual informed consent for this retrospective analysis was waived.

Open Access Statement: This is an Open Access article distributed in accordance with the Creative Commons Attribution-NonCommercial-NoDerivs 4.0 International License (CC BY-NC-ND 4.0), which permits the noncommercial replication and distribution of the article with the strict proviso that no changes or edits are made and the original work is properly cited (including links to both the formal publication through the relevant DOI and the license). See: https://creativecommons.org/licenses/by-nc-nd/4.0/.

\section{References}

1. Powell IJ, Bollig-Fischer A. Minireview: the molecular and genomic basis for prostate cancer health disparities. Mol Endocrinol 2013;27:879-91.

2. Topczewska JM, Postovit LM, Margaryan NV, et al. 
Embryonic and tumorigenic pathways converge via Nodal signaling: role in melanoma aggressiveness. Nat Med 2006; 12:925-32.

3. Mehlen P, Delloye-Bourgeois C, Chédotal A. Novel roles for Slits and netrins: axon guidance cues as anticancer targets? Nat Rev Cancer 2011;11:188-97.

4. Gonda Y, Andrews WD, Tabata H, et al. Robo1 regulates the migration and laminar distribution of upper-layer pyramidal neurons of the cerebral cortex. Cereb Cortex 2013;23:1495-508.

5. Legg JA, Herbert JM, Clissold P, et al. Slits and Roundabouts in cancer, tumour angiogenesis and endothelial cell migration. Angiogenesis 2008;11:13-21.

6. Mertsch S, Schmitz N, Jeibmann A, et al. Slit2 involvement in glioma cell migration is mediated by Robo1 receptor. J Neurooncol 2008;87:1-7.

7. Schmid BC, Rezniczek GA, Fabjani G, et al. The neuronal guidance cue Slit2 induces targeted migration and may play a role in brain metastasis of breast cancer cells. Breast Cancer Res Treat 2007;106:333-42.

8. Chang PH, Hwang-Verslues WW, Chang YC, et al. Activation of Robo1 signaling of breast cancer cells by Slit2 from stromal fibroblast restrains tumorigenesis via blocking PI3K/Akt/ $\beta$-catenin pathway. Cancer Res 2012;72:4652-61.

9. Mitra S, Mazumder-Indra D, Mondal RK, et al. Inactivation of SLIT2-ROBO1/2 pathway in premalignant lesions of uterine cervix: clinical and prognostic significances. PLoS One 2012;7:e38342.

10. Latil A, Chêne L, Cochant-Priollet B, et al. Quantification of expression of netrins, slits and their receptors in human prostate tumors. Int J Cancer 2003;103:306-15.

11. Choi YJ, Yoo NJ, Lee SH. Down-regulation of ROBO2 expression in prostate cancers. Pathol Oncol Res 2014;20:517-9.

12. Humphrey PA. Gleason grading and prognostic factors in carcinoma of the prostate. Mod Pathol 2004;17:292-306.

13. Pound CR, Partin AW, Eisenberger MA, et al. Natural history of progression after PSA elevation following radical prostatectomy. JAMA 1999;281:1591-7.

14. Tang H, Wei P, Duell EJ, et al. Axonal guidance signaling pathway interacting with smoking in modifying the risk of pancreatic cancer: a gene- and pathway-based interaction analysis of GWAS data. Carcinogenesis 2014;35:1039-45.

15. Biankin AV, Waddell N, Kassahn KS, et al. Pancreatic cancer genomes reveal aberrations in axon guidance pathway genes. Nature 2012;491:399-405.
16. Macias H, Moran A, Samara Y, et al. SLIT/ROBO1 signaling suppresses mammary branching morphogenesis by limiting basal cell number. Dev Cell 2011;20:827-40.

17. Dallol A, Da Silva NF, Viacava P, et al. SLIT2, a human homologue of the Drosophila Slit2 gene, has tumor suppressor activity and is frequently inactivated in lung and breast cancers. Cancer Res 2002;62:5874-80.

18. Dai CF, Jiang YZ, Li Y, et al. Expression and roles of Slit/Robo in human ovarian cancer. Histochem Cell Biol 2011;135:475-85.

19. Narayan G, Goparaju C, Arias-Pulido H, et al. Promoter hypermethylation-mediated inactivation of multiple SlitRobo pathway genes in cervical cancer progression. Mol Cancer 2006;5:16.

20. Dallol A, Morton D, Maher ER, et al. SLIT2 axon guidance molecule is frequently inactivated in colorectal cancer and suppresses growth of colorectal carcinoma cells. Cancer Res 2003;63:1054-8.

21. Parray A, Siddique HR, Kuriger JK, et al. ROBO1, a tumor suppressor and critical molecular barrier for localized tumor cells to acquire invasive phenotype: study in African-American and Caucasian prostate cancer models. Int J Cancer 2014;135:2493-506.

22. Ferrari MG, Ganaie AA, Shabenah A, et al. Identifying and treating ROBO1-ve /DOCK1+ve prostate cancer: An aggressive cancer subtype prevalent in African American patients. Prostate 2020;80:1045-57.

23. Zhou WJ, Geng ZH, Chi S, et al. Slit-Robo signaling induces malignant transformation through Hakai-mediated E-cadherin degradation during colorectal epithelial cell carcinogenesis. Cell Res 2011;21:609-26.

24. Yang XM, Han HX, Sui F, et al. Slit-Robo signaling mediates lymphangiogenesis and promotes tumor lymphatic metastasis. Biochem Biophys Res Commun 2010;396:571-7.

25. Shao Y, Zhou Y, Hou Y, et al. Prognostic implications of SLIT and ROBO1 expression in gallbladder cancer. Cell Biochem Biophys 2014;70:747-58.

Cite this article as: Kim SH, Kim TJ, Shin D, Hur KJ, Hong SH, Lee JY, Ha US. ROBO1 protein expression is independently associated with biochemical recurrence in prostate cancer patients who underwent radical prostatectomy in Asian patients. Gland Surg 2021;10(10):2956-2965. doi: 10.21037 /gs-21-406 